\title{
TRAPEZOIDAL AND APSIDAL RIBBED VAULTS SMART 3D RECONSTRUCTION
}

\author{
Capone M., Palomba D., Scandurra S., Lanzara E. \\ University of Naples Federico II, Italy \\ mara.capone@unina.it, daniela.palomba@unina.it,simona.scandurra@unina.it, emanuela.lanzara@unina.it
}

\section{Commission II}

KEY WORDS: Ribbed vaults, Smart 3D reconstruction, Semantic-driven analysis, Shape grammar, Procedural modelling.

\begin{abstract}
:
The automatic generation of structured 3D model from point clouds is a primary focus for a lot of researches in the area of 3D modelling and HBIM. The use of procedural modelling techniques, based on set of geometric rules, is spreading the construction of parametric objects and processes for survey data mapping. In this research field one of the most common methodological approach consists in testing different procedures based on use of specific data from point cloud. One of the main problem to solve is to define the best parameters to use in relation to geometric features of different architectural element and what are the data to extract from point cloud. Starting from shape grammar approach, the main goal of our research is to define 3D parametric models for ribbed vaults suitable to generate 3D smart model for different uses. Most researches in this area deal the case of rectangular or square plan vaults, we addressed the case of trapezoidal vaults and different rules used for apsidal vaults tracing. Our aim is to define an adaptive parametric model that we can use to generate a 3D ideal model or a mixed reality based model. In order to evaluate our tool we compared two 3D models with point clouds: the first is based on geometric rules from sources, the second is based on some specific data from point cloud. Testing the tool on two gothic churches in Naples, the church of S. Lorenzo and the church of S.Maria di Donnaregina, we are going to improve it.
\end{abstract}

\section{INTRODUCTION}

The automatic generation of structured 3D model from point clouds is a primary focus for a lot of research in the area of $3 \mathrm{D}$ modelling and HBIM (Dore, 2019)

The use of procedural modelling techniques, based on set of geometric rules, is spreading the construction of parametric objects and processes for mapping these library objects to survey data (Dore, 2019).

In this research field one of the most common methodological approaches consists in testing different procedures based on use of specific data from point cloud.

One of the main problem to solve in this process is to define the best parameters to use in relation to geometric features of different architectural elements and what are the data to extract from point cloud.

Starting from shape grammar approach (first introduced by George Stiny and James Gips in 1972) the main goal of our research is to define $3 \mathrm{D}$ parametric models for ribbed vaults suitable for different uses.

Based on semantic-driven analysis, we can decompose historical building into architectural elements that can be clearly identified. Each element is composed of different components that we have to define and we can use for a semantic classification. (Capone, 2021). In this paper we are going to present some results of wider research on ribbed vaults .

We have grouped the parts that compose ribbed vaults into main elements and components and we have defined different set of geometric rules used to generate each of them.

We defined set of geometric rules from comparison between different historical sources and cases study. Our aim is to define an adaptive model, an advanced procedural modelling tools, that we can use to generate a $3 \mathrm{D}$ ideal model or a mixed reality based model.

We can also use this tool to generate 3D smart model for different uses, for Cultural Heritage dissemination, for virtual reality application and for HBIM.

\section{RELATED WORK}

In recent years, one of the main focus of smart 3D reconstruction is automated processes definition based on sets of geometric rules and semantic driven analysis.

The wide spread of computational tools allows us to test methods based on comparison between "ideal model" from historical sources and 3D model based on some specific geometrical inputs from point-cloud data (Angjeliu 2019).

There are a lot of researches that describes applications used to model classical architectural elements (Dore, 2019), column, barrel vaults, groined vaults and so on.

Some software houses are implementing the BIM software with specific parametric objects usable for historical building modelling. Edificius ACCA Software, for example, provides a dedicated environment for HBIM modelling including some specific tools to model barrel vaults, groin vaults, pavilion vaults, domes and the main vaults that can be generated by surfaces intersection. There are not specific tools for gothic vault tracing. However, it is only possible to use pointed arch to model these types of vaults.

There is a lot of research in progress that deals with ribbed vaults parametric model generation (Angjeliu 2019) and test this tool for ribbed vault geometry study (Bagnolo 2021).

Most researches in this area (Webb, 2019) deal the case of rectangular or square plan vaults, we are going to address the 
case of trapezoidal vaults and to analyse different rules used for apsidal vaults tracing .

\section{DEVELOPED METHODOLOGY}

There was not only one method for ribbed vaults tracing but the construction of ribbed vaults is based on a set of geometric rules that designers used to control the building process (Buchanan, 2021). Starting from the main rules applicable to most of the case studies we are going to define an adaptive parametric tool for gothic vault modelling. In order to evaluate the best parameters to use we have to compare the theoretical rules with the real buildings to define a general scheme. In this paper we present a part of a wider research on ribbed vaults related to trapezoidal and apsidal vaults 3D modelling.

From a methodological point of view the main steps of our research are:

1. geometric rules and parameters definition from historical sources;

2. parametric model definition for procedural modelling;

3. test on different case studies, 3D modelling using some data from point cloud,

4. Review process of the parametric tool.

The workflow based on the computational approach, that we are testing in our research, includes the main following activities:

1) vault parametric models based on treatise rules; parametric object oriented model;

2) data acquisition (point cloud from TLS or photogrammetry);

3) overlapping of parametric object oriented model on point cloud;

4) critical interpretation of data (geometric configuration hypothesis);

5) construction of the realty based model;

6) construction of an interoperable geometric model to be used both as an interface for $3 \mathrm{D}$ communication system and for HBIM system.

Generally, this process is based on knowledge and analytical and critical abilities of individual scholars, the idea is to increase the efficiency of the system through collaborative workflow forms that allows you to optimize the processes through effective knowledge management actions (Capone, 2019).

\section{GEOMETRIC RULES FROM SOURCES}

The ribbed vaults consist in a framework of ribs or stone arches, upon which the real vaults rest. These vaults are usually constructed of a lighter material than the ribs upon which they rest, and between which they constitute, in fact, a kind of thin panel (Willis, 1842). The construction of these ribs offers no difficulty in stone-cutting, each can be separately considered, a simple arch; nevertheless, the forms of its voussoirs can scarcely have been obtained without tracing on the ground the full-sized shape of this arch, from which to obtain the face moulds of the voussoirs (Willis, 1842).

Starting from historical sources and study of Gothic Cathedrals we have defined groups for indexing different apses in relation to plan geometry tracing. We have defined four typologies based on use of circle, dodecagon, decagon and octagon (Figure 1). We used the same methodological approach for quadripartite ribbed vaults. First, we grouped the ribbed vaults in relation to plan shape that can be square, rectangular or trapezoidal and we have defined different geometric rules for tracing.

Our aim is to define the best parameters to use to obtain an adaptive parametric model in which there are simple parameters to modify in order to generate the different typologies of vaults that we have defined.
We are improving our parametric models using some different rules from treaties of Viollet-le-Duc, Choisy, Willis and by comparison with existing buildings. The wide spread of specialist survey techniques, such as photogrammetry and laser scanning, allow us to analyse many examples from which general rules might be deduced.

It is only by comparing many examples that this can be done, general rules deduced from single instances are commonly worthless. Ruins afford the most valuable information upon construction. The best instructor of all, perhaps, is a building which is being pulled down. In ordinary cases, the upper surfaces of the vaults are so often covered with courses of rubble and concrete rubbish and filth, and the lower surfaces with whitewash and paint, that when every facility has been obtained for examination, the jointing of the masonry and actual construction of the vault will still remain an unfathomable mystery (Willis, 1842). The knowledge of the building methods would greatly assist us in the process of digital twin generation.

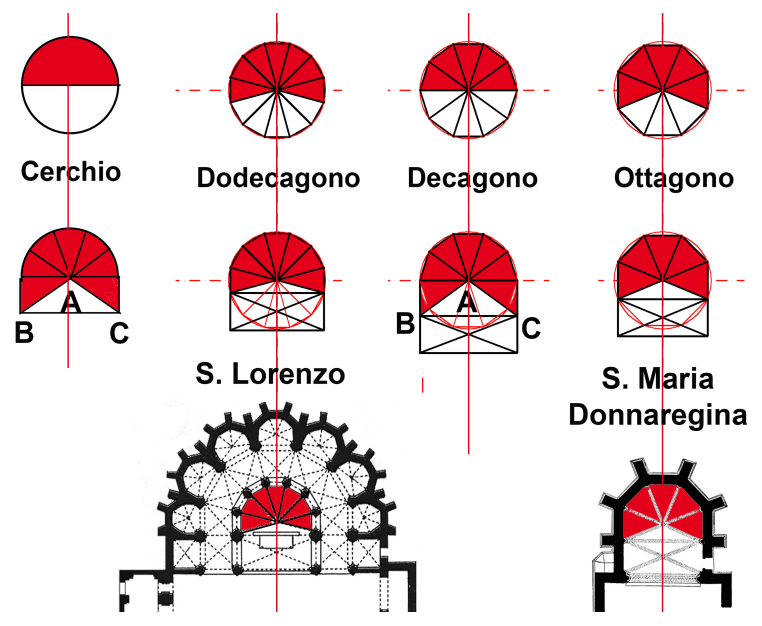

Figure 1. Apse tracing: geometric rules for adaptive parametric model definition - case studies in Naples

\subsection{Quadripartite trapezoidal ribbed vaults tracing}

The first step for ribbed vaults tracing is the plan design. The ribs layout it is very simple if the plan is square or rectangular but it is more complex if the plan is trapezoidal.

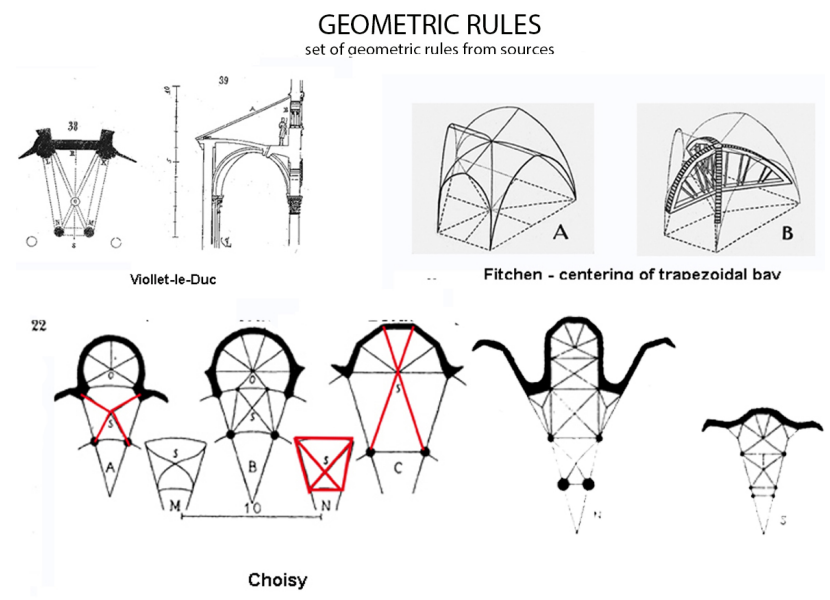

Figure 2: trapezoidal vault tracing: different geometric rules for plan tracing from sources 


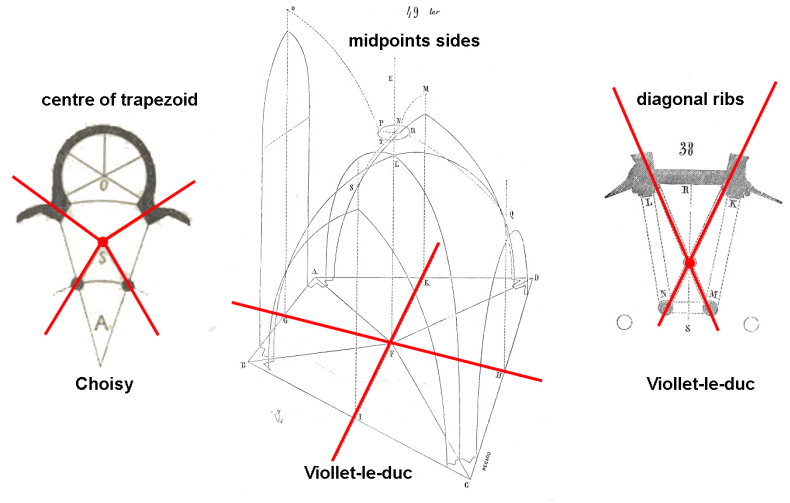

Figure 3: trapezoidal vault tracing: geometric rules from sources
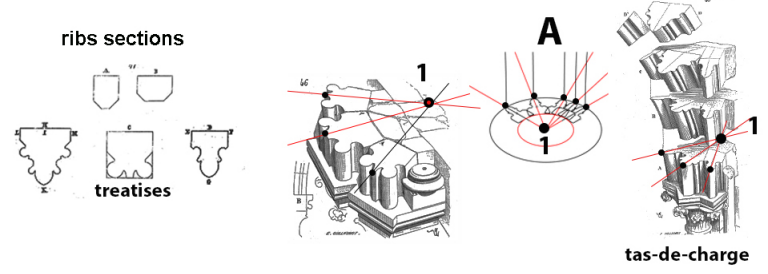

Figure 4: Tas de charge: geometric rules from sources

In fact, in the first case there is only one geometric rule to ribs design, they must be diagonal, on the contrary if the plan is trapezoidal the ribs can be the diagonal of the trapezium or they can be generated using different rules (Figure 2).

The ribs can be diagonal, they converge on the centre of the trapezoid - centre ribs (Choisy 1841 - 1909) - or they can be defined using the intersection point between the lines that linked the midpoints of the opposite sides of trapezoid - midpoints ridges (Viollet-le- duc 1854 - 1868 and Fitchen 1961).

If the ribs are diagonal there are two different rules: the diagonal arches are two circle arches or they are composed by four different arches. In the first case the arches are continuous and the main keystone altitude is lower than the perimetral arches keystones. In the second case the main keystone and the perimetral arches keystones are at the same height, for this reason the diagonal arch is composed by two arches that are different, the result is that the diagonal arches are not continuous (Figure 2).

The second step is to define the geometric rules used for tracing aches and ribs. All these arches are usually semicircular, and their crowns are all placed at the same height, to allow for which they are made to spring from different levels. Starting from Villard rule and from Viollet-le-duc Dictionnaire we have defined the main rules to use to generate an adapt geometric model in which all the arches have the same curvature (Villard rule). The radius can be immediately defined if the ribs are diagonal (continuous arches) and the perimetral arches will be obtained using the same radius. In this case we can modify the impost line in relation to the crown line, the ridge, that can be straight, inclined or horizontal, or curved.

When the ribs are not continuous diagonals, or they are centre ribs or midpoints ribs, there is not a single rule to trace them and the perimetral arches (Figure 3). Starting from the main keystone height we can define the keystone height of the perimetral arches in relation to the crown line shape. Also, in this case it can be straight, horizontal or inclined, or curved.

In order to define the geometric building process we have to generate the extrusion lines, that are the ideal rails along with the voussoirs moves to generate each rib, all these lines are the so called wireframe model. We have to define from sources a library of the main sections of the voussoirs used for ribs construction and the ribs spring. The ribs can be independent each other, in this case the voussoirs are trimmed when necessary or the ribs are joined together and there is the so called tas de charges (Figure 4).

\subsection{Apses tracing}

The traditional semi-circular apse, greatly enlarged and varied in the developed style by a polygonal form, is the characteristic eastern termination of gothic churches. The early Gothic apses plan are usually semi-circular, they are generally divided in five cells, whose ribs intersect upon the first transverse rib of the choir. This tripartite compartment and the semicircle compartment together may be considered one octopartite vault. Starting from semi-circular plan the apse tracing can be based on some different polygon geometry such as octagon, decagon and dodecagon (Figure 1). We can define the elements witch the apse is composed: the radial arches, the perimetral arches and the main arch BC (Figure 1). In the case of octagon and dodecagon the radial ribs converge in the centre of the circumscribed circle and they are all the same. In the case of semicircle and decagon the ribs $\mathrm{AB}$ and $\mathrm{AC}$ are different (Figure 1). The perimetral arches can be traced in different ways, they are usually pointed arches, equilateral, lancet or depressed, but they could be circle arches, elliptical arches or some different kind of arch. The keystones height is generally the same of the main keystone for this reason the ridge is straight. Starting from this geometric rule generally used we can define the impost line height, A, in relation to the main keystone height, $\mathrm{H}$. F is a parameter that we can obtained in relation to arch typology and $\mathrm{A}=\mathrm{H}-\mathrm{F}$ (Figure 6).
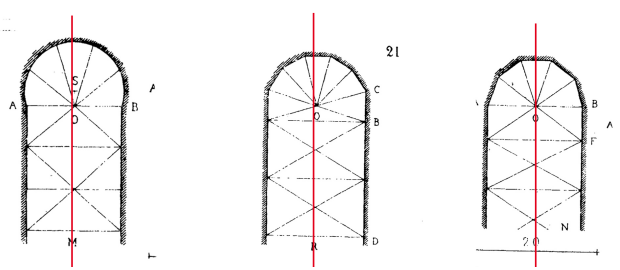

Figure 5: Apses tracing - Choisy 1889

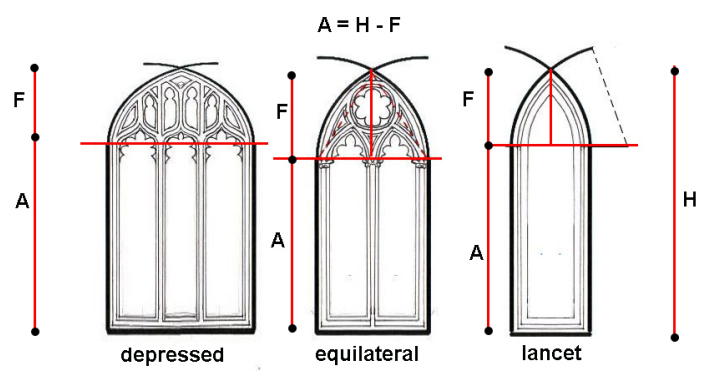

Figure 6: some geometric rules for perimetral arches tracing

\section{PROCEDURAL MODELING}

We present an approach for automatic modelling of the trapezoidal and apsidal ribbed masonry vaults based on a parametric model. Our aim is to define an algorithm for smart 3D model able to reduce time-consuming for modelling activities in order to generate an approximation of the real vaults. The workflow based on the computational approach, that we are testing in our research, includes the main following activities: 1) vault parametric models based on treatise rules; parametric object oriented model; 2) data acquisition (point cloud from TLS or photogrammetry); 3) overlapping of 
parametric object oriented model on point cloud; 4) critical interpretation of data (geometric configuration hypothesis); 5) construction of the realty based model; 6) construction of interoperable geometric model to be used both as an interface to a 3D communication system and as part of a HBIM system. Generally this process is based on knowledge and analytical and critical abilities of individual scholars. The idea is to increase the efficiency of the system through collaborative workflow forms that allows you to optimize the processes through effective knowledge management actions.

The workflow for parametric model definition can be summarized in these main steps:

1) vault plan definition;

2) extrusion lines definition based on treatises geometric rules, wireframe 3D model;

3) Tas de charge parametric definition;

4) 3D model generation using extrusion lines from literature;

5) Web surfaces generation.

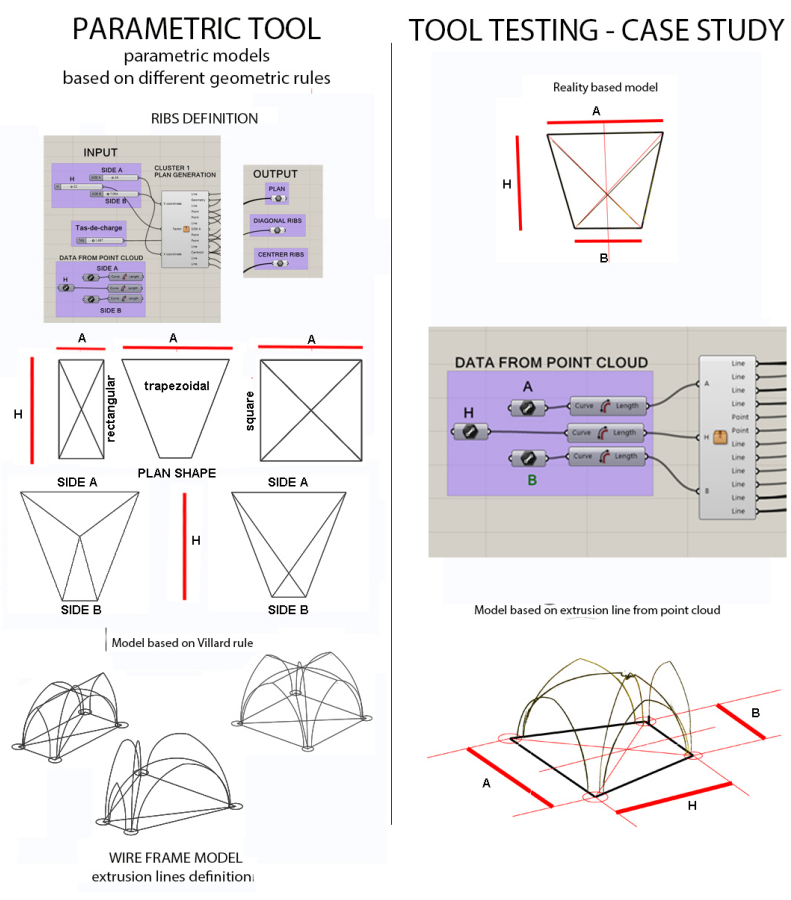

Figure 7: CLUSTER 1 and CLUSTER 2 Parametric tool for automatic modelling of quadripartite, from square, rectangular to trapezoidal vault.

As we noted there are different methods to define ribs curvature. Starting from Villard rules (the curvature is the same for all the ribs) to more complex rules. We have defined the fixed elements and variables to generate a parametric model based on treatises drawings. We generated a simplified model without ribs and arches thickness, without web surfaces between ribs and arches. This wireframe model is composed by lines of the ribs: moving the section ribs along these lines (extrusion lines) we can modelling the ribs (Figure 7).

The variables data are: 1. the ridge configuration (straight or curve), the ribs curvature, that can be generate using different geometric methods, 2. the starting point location of the extrusion lines, the shape and the spring points of the ribs, that can be located at different levels in relation to the impost plane of the vault. In our model changing the input parameters we can generate different models based on different geometric rules. For example, using Villard rule, staring from diagonal arch we.
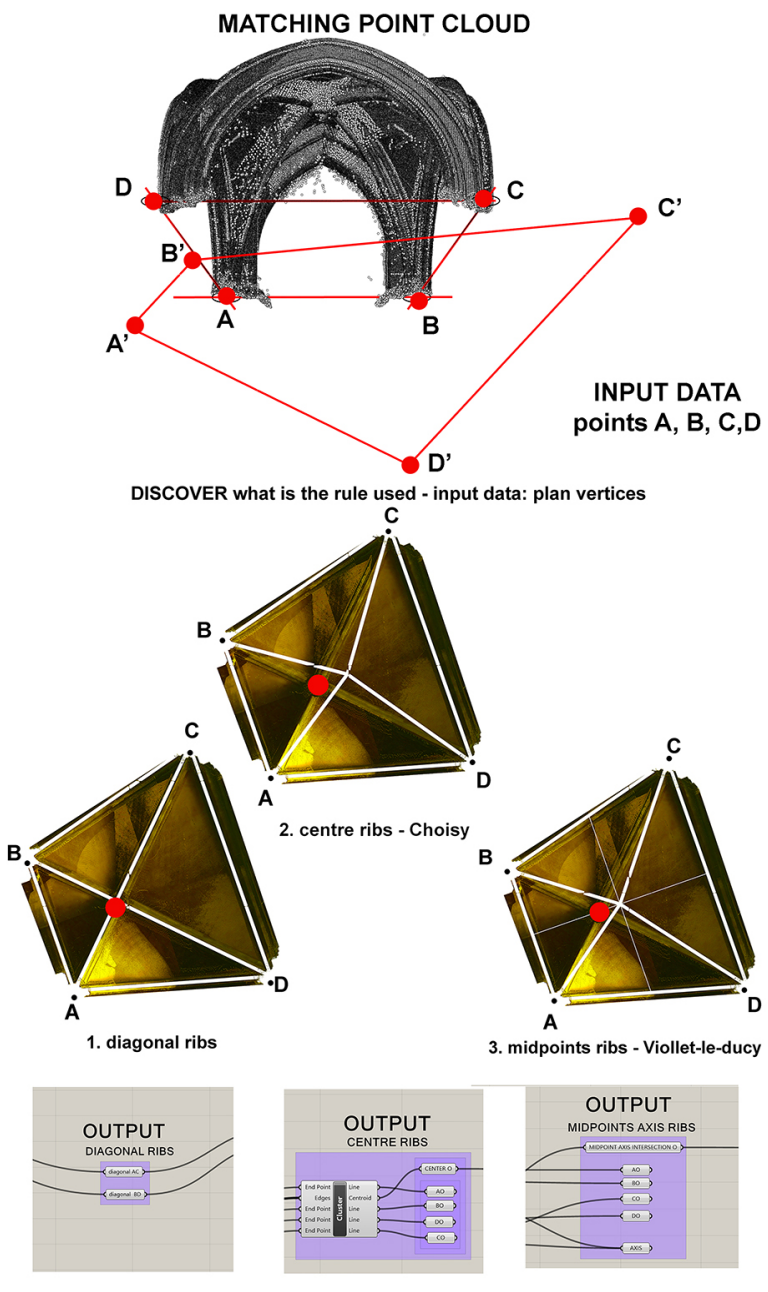

Figure 8: Matching point cloud trapezoidal quadripartite vault church of S. Lorenzo for plan tracing.

can define the curvature used for all ribs and we can generate two different models: one for straight ridge hypothesis and the other for curved or inclined ridge

If the ridge is straight, the tool are able to automatically determinate the spring points of all elements, ribs, wall arches and transverse arches.

In this case the spring points are not on the impost plane of the vaults and each of them is located at a different height in relation to the horizontal ridge. Overlapping these two models on point cloud we can evaluate if the designer used the Villard rule or not, in this case we can formulate another design hypothesis to match with ideal models generated using other geometric rules.

\subsection{Quadripartite trapezoidal ribbed vaults tracing}

The first step for trapezoidal ribbed vault design is plan definition, the projection on the horizontal plane of the ribs. These segments are the traces of the vertical planes where the ribs are located. They can be overlapped with diagonals of trapezoid, they can converge in the trapezoid centre or they can converge in the intersection point of midpoints lines.

We have done a parametric model that allows you to modify the trapezoid shape using three parameters: the side A, the side B and the height $\mathrm{H}$ (Figure 7). 


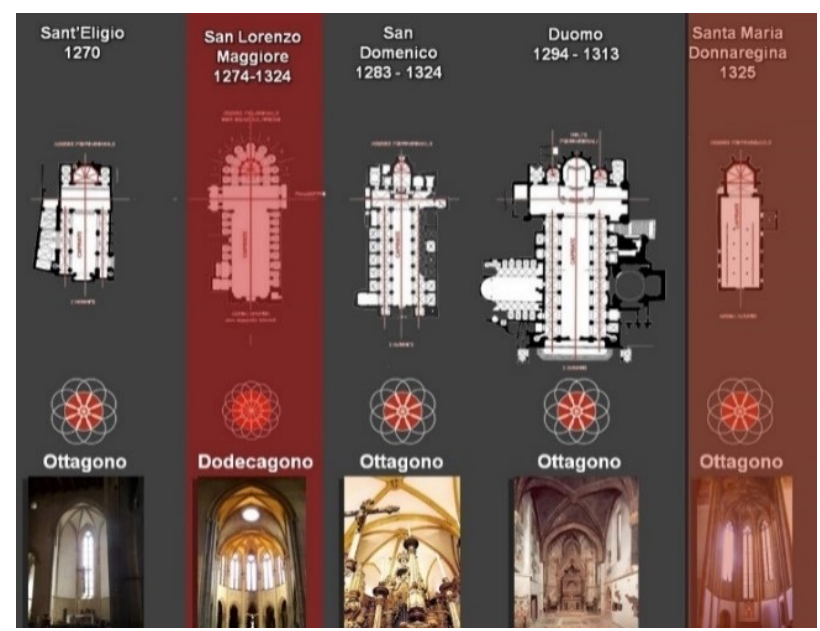

Figure 9. mapping Gothic in Naples.
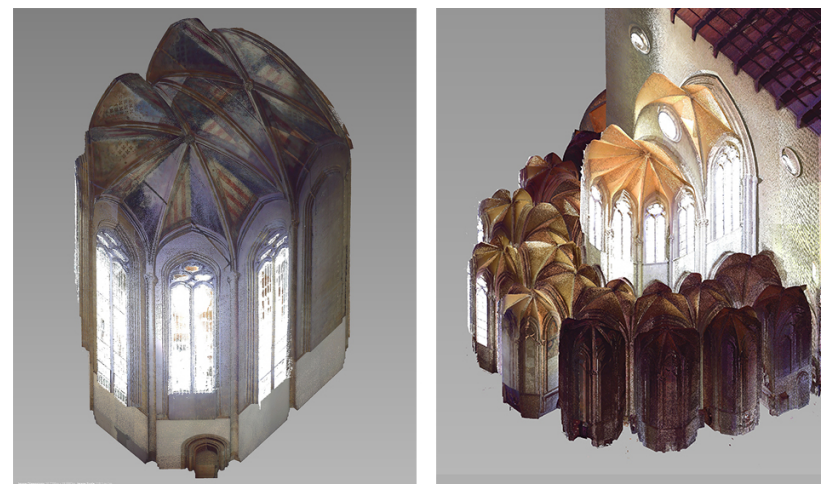

Figure 10. Data acquisition - S. Lorenzo and Donnaregina point cloud from laser scanner

The output of the CLUSTER 1 are three different configurations for plan ribs distribution: 1.diagonal ribs, 2.centre trapezoid ribs, 3. midpoints ribs.

For each of them we have defined a parametric model able to generate extrusion line for arches and ribs modelling.

CLUSTER 2 allows you to automatically generate two different wireframe model: one is based on Villard rule, all arches with the same curvature, and the other is based on horizontal ridge configuration, in this case the main keystone is at the same altitude of the perimetral arches keystones.

There in not only one rule for ribs curvature, for this reason the best way to generate a smart 3D model that best fit the real one is used some geometrical inputs based on specific measurements. The input data from point cloud are the main keystone altitude and the perimetral arches keystones altitude.

\subsection{Apses tracing}

Starting from Choisy layout we have defined an adaptive parametric model based on geometric rules. Our parametric model allows you to generate these types of apses: semicircular, based on octagon, decagon and dodecagon.

The first part of the parametric model algorithm is used for automatic generation process of the extrusion lines.

The INPUT data are the number of the sides of the generator polygon, the OUTPUT are the plan configuration, the projection of perimetral arches, radial arches and the main arch and 3D model of these arches, the wireframe model.

The arches are generated using one of the main common rule: the radial arches are composed by half circle arch and the main arch is a circle arch.
Our tool allows you to define different kind of pointed arches that can be used for perimetral arches generation. Changing the centre we can get a lancet pointed arch, an equilateral pointed arch or a depressed pointed arch (Figure 6). If the ridges of the radial vaults are horizontal we know that the main keystone of the apses vault and the keystones of perimetral arches are at the same height. The tool is automatically able to generate the perimetral arches. The INPUT data is the arch typology, from this data we can define $\mathrm{F}$ length and $\mathrm{A}=\mathrm{H}-\mathrm{F}$. (Figure 6).

\section{TOOL TESTING ON CASE STUDIES}

We tested our tool for apsidal and trapezoidal vaults smart 3D modelling on gothic churches in Naples. We are mapping Gothic churches in Naples in relation to apse shape and we have chosen two case studies, S. Lorenzo based on dodecagon and Donnaregina based on the octagon geometry (Figure 9).

Gothic architecture spread in Naples when Charles I of Anjou came to Naples in 1266. We need to compare the gothic architecture in Naples with original model to understand it, thus we have to know the main feature of French Gothic architecture. Therefore, our parametric model is based on French sources. Starting from Villard de Honnecourt, our aim is to improve it by comparison between ideal models and real buildings.

Matching the parametric model on point cloud data we can discover new geometric rules or to understand what is the rule used (Figure 8).

Starting from labelling process on point cloud from laser scanner data, we are going to show 3D modelling process for trapezoidal and apsidal vaults. In order to evaluate our tool we have compared two 3D models with point clouds: the first is based on geometric rules from sources, IDEAL MODEL, the second is based on some specific data from point cloud, REALITY BASED MODEL. The comparison allows us to improve the tool.

\subsection{Adaptive model for realty based models generation: trapezoidal vault}

We have defined an algorithm for reality based model generation for trapezoidal vaults and for different kind of apses. The first step of the process is data acquisition and the sematic segmentation of point cloud that allows you to define the architectural elements. The goal is to transform a point-based representation into a parametric (semantic) model (Croce 2021). In this paper we are going to show the process used for trapezoidal vaults and apses vault model.

The INPUT data for trapezoidal vault are plan vertices ABCD the OUTPUT of the first algorithm of our tool are three different configurations for ribs plan tracing: 1.diagonal ribs, 2 . centre trapezoid ribs, 3. midpoints ribs. We can discover the rule used by overlapping planes ribs layout generated on point cloud orthogonal plan view. In our case study, S. Lorenzo trapezoidal vault, the ribs are diagonal (Figure 8).

We can use two different process to generate extrusion lines: the first is based on extrusion lines from point cloud data (Figure 11 ), in this case the tool allows you to transform the section from point cloud into the best curve that fit the real ones, the second is based on some specific measurement (end points and spring points of ribs extrusion lines and keystones height), the tool automatically generate the arches. In order to define the geometric shape of the extrusion lines we used more than one sections from point cloud. The comparison allow us to define the curves nearest to the real ones. The main steps for ribs tracing based on point cloud data are (Figure 11): 
ADAPTIVE PARAMETRIC MODEL

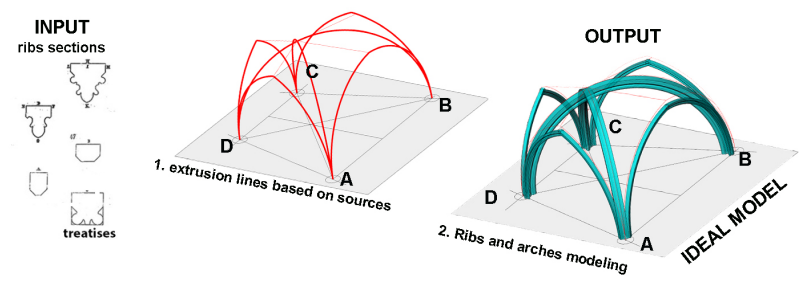

POINT CLOUD DATA
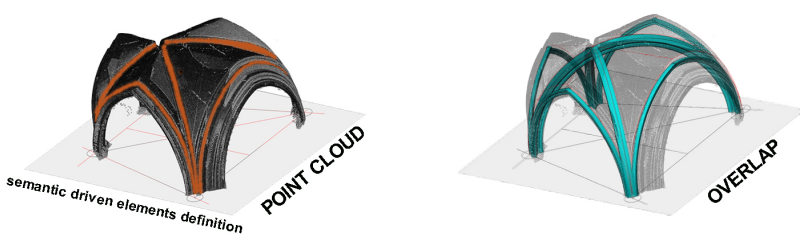

COMPARE
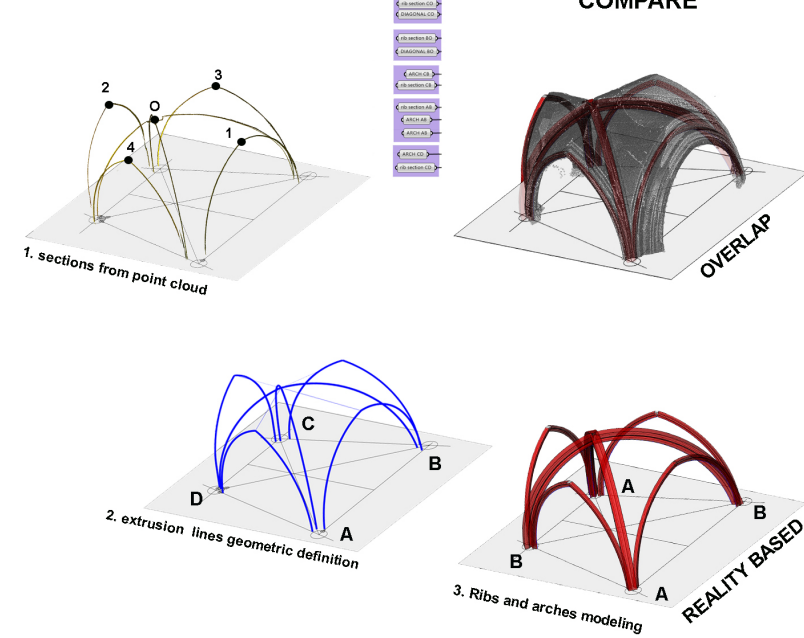

Figure 11. Tool test for trapezoidal Church of S. Lorenzo vault. Comparison between ideal model and reality based model

1) Point cloud section using vertical planes,

2) Extrusion line geometric definition from point cloud data;

3) $3 \mathrm{D}$ ribs modeling using specific ribs section (profile curve).

The problem to solve in transformation process from point cloud data to geometry definition of extrusion lines is to find the curves that best fit the real ones. From theoretic framework we know that these curves can be composed by only one arch, one arch and a vertical straight line, an oval arch or an elliptical arch. In order to search the best geometry for each rib and perimetral arches of the vault we are testing some processes based on the use of evolutionary solver and points on point cloud sections.

The other method for ribs arches tracing, arches method, is based on keystone height from point cloud and spring line.

The centre of the arches are defined as intersection between the perpendicular line in the midpoint of the chord with impost line. Overlapping the arches automated generate using this method we can observe that the curves are sometime different from the real one.Testing the arches method for diagonal ribs tracing of .S. Lorenzo trapezoidal vaults the result is: diagonal arches are not continuous, they are not circular arches, the perimetral arches $\mathrm{BC}$ and $\mathrm{AD}$ are pointed arches and the perimetral arch DC is a pointed arch with a straight segment.

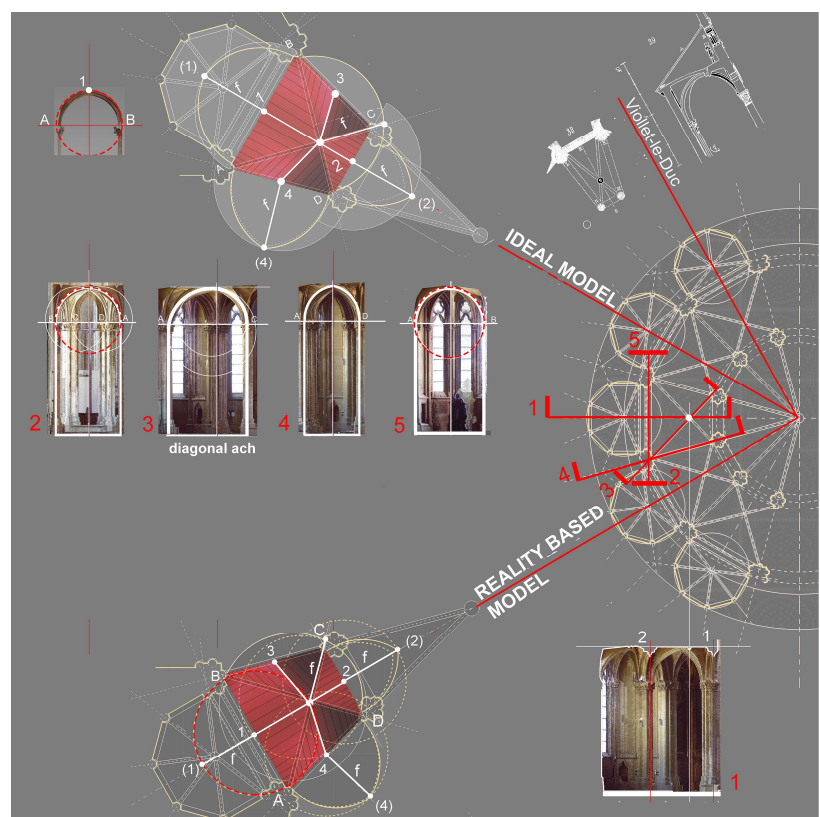

Figure 12. Comparison between ideal model and reality based model

Overlapping the arches generate using the aches method we can see that the arches $\mathrm{BA}, \mathrm{CD}$ and the diagonal arch $\mathrm{DO}$ are perfectly overlapped, but the diagonal arch $\mathrm{BO}$ and the perimetral arch DC are very different in relation to the point cloud section.

At the end of the process we have to choose the 3D model Lod (level of detail) in relation to our needs, LOIN (Level Of Information Need).

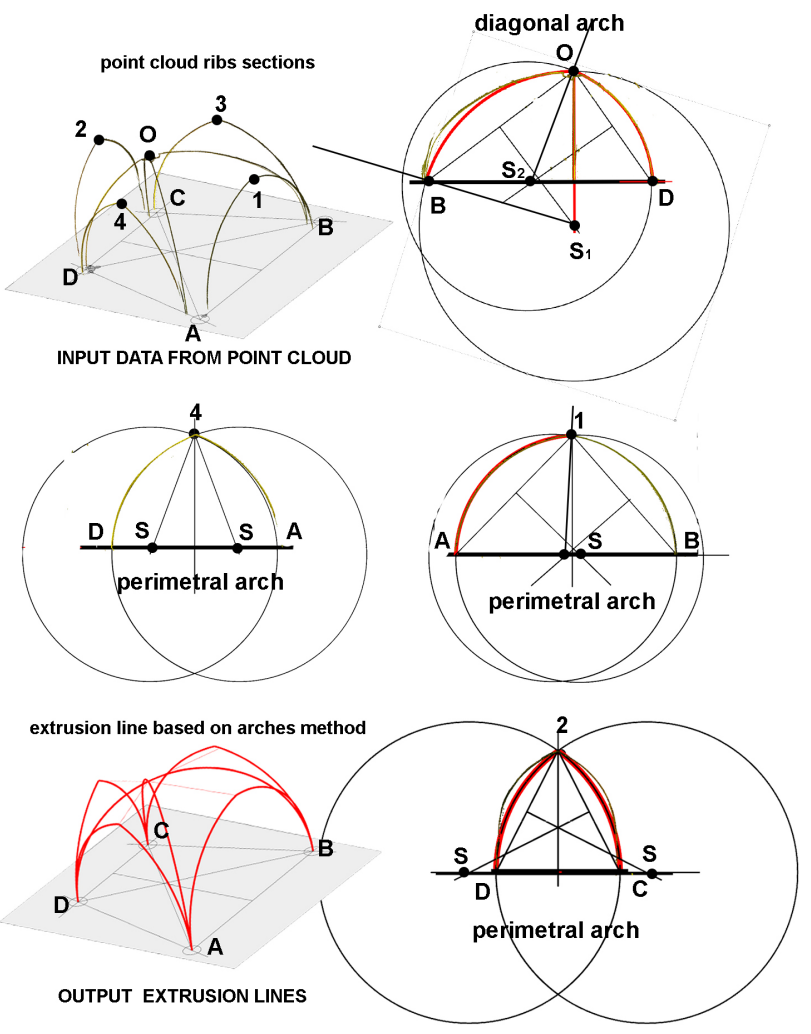

Figure 13. Church of S. Lorenzo trapezoidal vault: extrusion lines tracing based on arches method. 


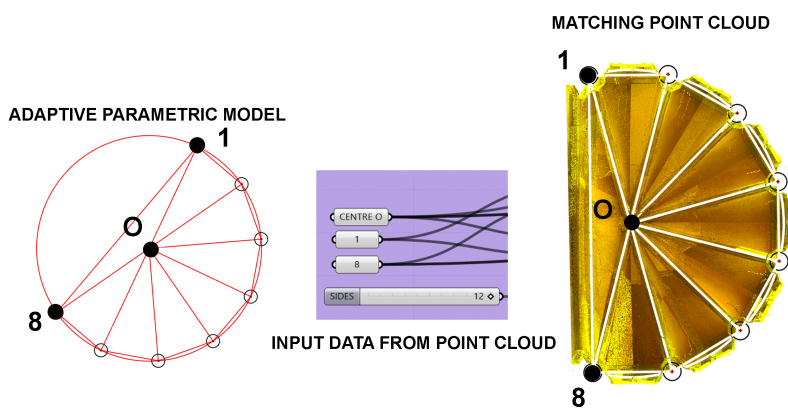

Figure 14. Church of S. Lorenzo apses vault: matching the adaptive model on point cloud

\subsection{Adaptive model for realty based models generation: apses tracing}

The geometric rules used for apses tracing allows you to generate a $3 \mathrm{D}$ model that is generally very close to the real one. We tested the tool based on these rules for dodecagonal apses of the church of S. Lorenzo and octagonal apses of the church of Donnaregina.

The first step of the process consists in matching the adaptive parametric model on point cloud. In order to match our parametric model on point clouds of S. Lorenzo apses the INPUT data are the centre $\mathrm{O}$, the sides number of the polygon and the main arch 18 for Church of S. Lorenzo (Figure 14). We automatically get the plan and the wireframe model of the apses vault (OUTPUT). We can model the ribs by choosing the ribs sections and different configurations of tas de charges (Figure 4). Our IDEAL MODEL is based on Villard rule: all the arches with the same curvature.

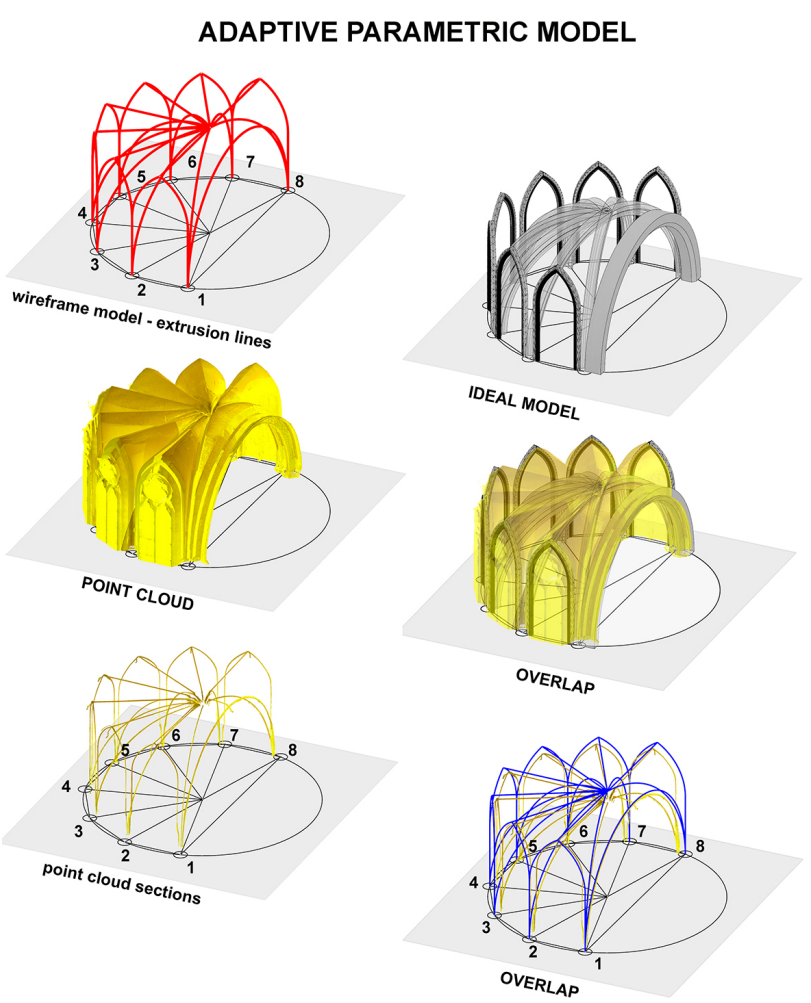

Figure 15. Church of S. Lorenzo apses vault: compare between reality based model and ideal model

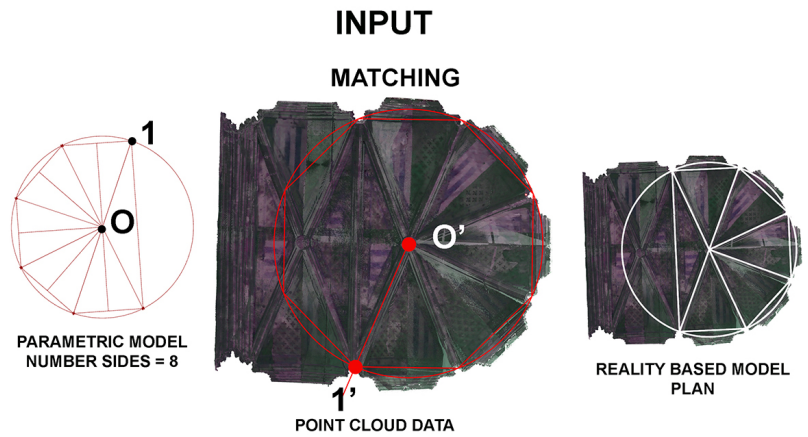

Figure 16. Church of Donnaregina apses vault: matching the adaptive model on point cloud.

The perimetral arches are equilateral pointed arches and the ridges are horizontal.

We can compare this model with point cloud ribs section and point cloud to evaluate the LOD of 3D model.

In the case of S. Lorenzo the ideal model is very close to point cloud therefore we do not need to define the model based on extrusion lines from point cloud sections.

We have tested the adaptive parametric model on Donnaregina octagonal apses using some different parameters to match ideal model on point cloud. We used the centre $\mathrm{O}$ and the radius of circumscribed circle, the point 1 .

Comparing 3D smart model with point cloud we can see that the ideal model fit the real one (Figure 18). All the radial arches are circle arches, the main arch is a pointed arch with the same curvature and the perimetral arches are equilateral pointed arches. We do not need to use the point cloud section to define the extrusion lines for ribs modelling.

\section{CONCLUSIONS}

The inputs data of our adaptive model are: plan size (vertices from point cloud), extrusion lines (from geometric rules or from point cloud data), extrusion lines localization, ribs and arches cross section, the ridge geometry (that can be slightly horizontal, inclined or curved) and the keystones configuration. We are going to test our algorithm for some different case studies to evaluate and to improve the tool. We are testing the use of evolutionary algorithms in order to define the extrusion line geometry from point cloud and in order to evaluate what is the set of geometric rules that best fit the case study.

\section{OUTPUT}

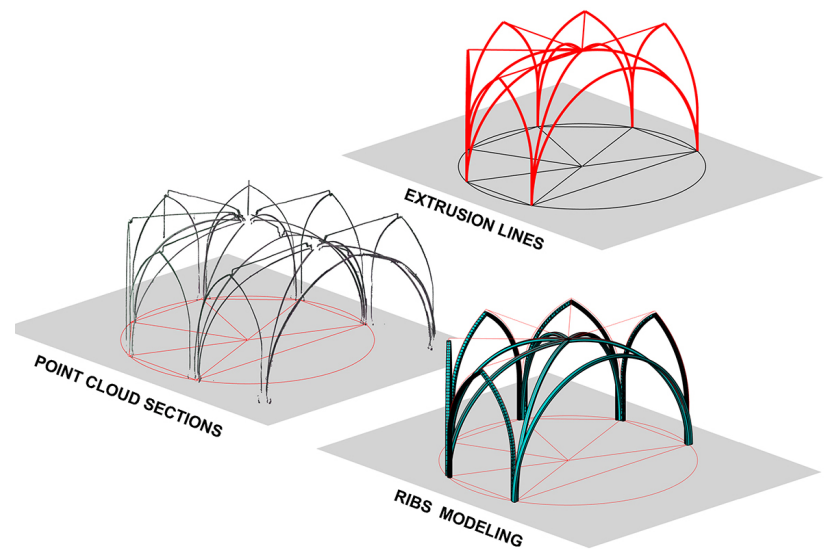

Figure 17.Donnaregina apses vault: automatic ribs modeling. 


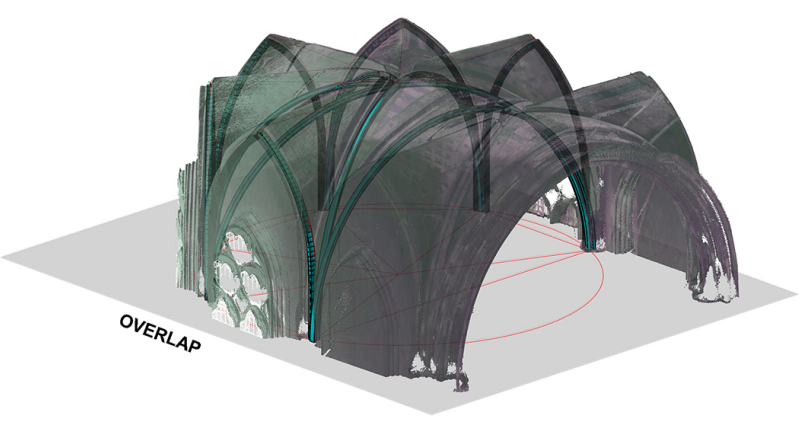

Figure 18.Church of Donnaregina apses vault: overlap ideal model on point cloud

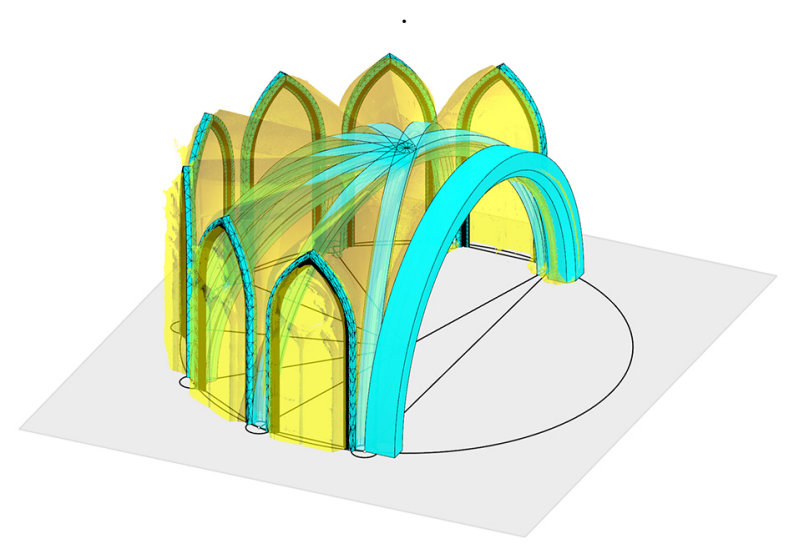

Figure 19. Church of S. Lorenzo apses vault: overlap ideal model on point cloud

Another goal of our research is to test the use of our parametric tool in HBIM environment.

We are going to test the interoperability between our procedural VPL tools (Grasshopper, Rhino) and Edificius, ACCA software, that allows us to model different kinds of vaults using parametric objects.

\section{REFERENCES}

Angjeliu, G., Cardani, G., Coronelli, D., 2019: A parametric model for ribbed masonry vaults. Elsevier. Automation in Construction. Volume 105.

Angjeliu, G, Cardani, G., Coronelli D., 2019. Digital modelling and analysis of masonry vaults. ISPRS. XLII-2/W11, pp 83-89

Bagnolo V., Argiolas, R., Vanini C, 2021. Algorithmic Modelling as a Key Tool for Ribbed Vault Geometry Nexus Network Journal - July 2021

Buchanan, A., Hillson, J., Webb, N., 2021.Digital Analysis of Vaults in English Medieval Architecture: Tracing English Gothic Vaults. New York and London. Routledge

Capone, M. and Lanzara, E.: 2021. PARAMETRIC LIBRARY FOR RIBBED VAULTS INDEXING, ISPRS, XLVI-M-12021, 107-115
Capone, M. and Lanzara, E., 2019. Scan to BIM vs 3D ideal model. HBIM: parametric tool to study domes geometry, ISPRS, XLII-2/W9, 219-226.

Calvo-López J., 2020. The geometry of rib vault in early modern Iberian and French literature. Stereotomy: Stone Construction and Geometry in Western Europe 1200-1900 Springer Nature 2020

Croce, V., Caroti, G., De Luca, L., Jacquot, K., Piemonte, A. and Véron, P., 2021. From the Semantic Point Cloud to Heritage-Building Information Modeling: A Semiautomatic Approach Exploiting Machine Learning. In: Remote Sensing 13(3):461

Choisy, A., 1841-1909. Histoire de l'architecture. Tome 2 https://gallica.bnf.fr/ark:/12148/bpt6k6584016q/f305.item\#

Dore C. Maurice Murphy, 2019. Historic Building Information Modelling (HBIM). In Architecture and Design: Breakthroughs in Research and Practice vol 2. IGI Global.

Fitchen, J., 1961. The construction of Gothic Cathedrals. A study of Medieval Vault Erection. The University of Chicago Press

Maira Vidal, R., 2015: The construction of sexpartite vaults in Europe. 5ICCH proceedings, Vol. II : 533-540. ISBN: 978-1329-15031-7.

Webb, N., Buchanan, A., 2019. Digitally aided analysis of medieval vaults in an English cathedral, using generative design tools International Journal of Architectural Computing, vol. 17, 3: pp. 241-259.

Webb, N., Buchanan, A., 2019..Digitally aided analysis of medieval vaults in an English cathedral, using generative design tools International Journal of Architectural Computing, vol. 17, 3: pp. 241-259.

Willis, R., 1842. On the construction of the vaults of the Middle ages. In: Transaction of the Royal Institute of British Architects of London. Vol. I, Part II. London: Longman, Brown, Green, and Longmans. http://robertwillis2016.org/Library/pdf/1842_Willis_The_constr uction_of the_vaults_of_the_Middle_Ages_CAL_OCR.pdf 